\title{
A Simple Method to Quantify Glycogen from Human Platelets
}

\author{
Débora Santos Rocha', Samir Khal de Souza1, Tor Gunnar Hugo Onsten², Roselis Silveira Martins da Silva ${ }^{3}$ and Marcos Emílio Frizzo,,2 \\ ${ }^{1}$ Department of Morphological Sciences, Universidade Federal do Rio Grande do Sul, Brazil \\ ${ }^{2}$ Hemotherapy Service, Clinical Hospital of Porto Alegre, Universidade Federal do Rio Grande do Sul, Brazil \\ ${ }^{3}$ Department of Physiology, Universidade Federal do Rio Grande do Sul, Brazil
}

\begin{abstract}
Objective: The aim of our study was to establish a protocol to quantify the glycogen from human platelets. Methods: The total glycogen was determined in the platelet concentrate (PC) obtained from healthy male volunteers. The assay comprised the following steps: (1) cell lysing and glycogen extraction, (2) precipitation of glycogen and its washing, (3) conversion of glycogen to free glucose, (4) neutralization, and (5) detection of free glucose. Results: In our analysis, the measured glycogen content in the $P C$ was $0.263 \pm 0.101 \mu \mathrm{g} / 10^{6}$ platelets (mean $\pm S D, n=16$ ) Discussion: Despite the potential importance of glycogen determination in the platelets, a biochemical method to quantify this polymer in these cells is not available at the moment. Our protocol proved to be a simple method to measure these reserves from a blood sample. We expect to contribute to future studies of platelet metabolism, especially regarding glycogen function in these cells.
\end{abstract}

\section{Keywords: Alzheimer's Disease; GSK3 $\beta$; Depression; Diabetes}

Abbreviations: ADP: D-Hexose-6-Phosphotransferase; GS: Glycogen Synthase; GP: Glycogen Phosphorilase; PRP: Platelet-Rich Plasma; PC: Platelet Concentrate; G: Glycogen; $\mathrm{G}_{\mathrm{s}}$ : Glycogen Concentration Standard; Abs: Absorbance of Glucose Standard; Abs: Absorbance of Glucose from the Sample; V: Total Volume of Glycogen Sample; N: Total Number of Platelets; GSK3 $\beta$ : Glycogen Synthase Kinase 3- $\beta$

\section{Introduction}

Glycogen is abundant in the platelet cytoplasm, where it serves as a major energy source for a variety of functions, including the release reaction $[1,2]$. In electron micrographs, platelet glycogen appears as individual particles, randomly distributed in the cytoplasm as discrete masses of uniform size [2]. The glycogen particles seem not to exist in isolation, but are associated with enzymes involved in glycogen synthesis and degradation $[3,4]$. This macromolecular structure seems to be a complete functional unit in the cell, and according to some authors, should be considered an organelle, called the glycosome [1]. More recently, these structures were described as bounded by a membrane and filled with particles of glycogen [5]. Another structure present in platelets, the a-granule, may occasionally contain glycogen and may be confused with glycosomes. The reason for this co-localization in these organelles is unknown [5].

The existence of large amounts of glycogen and the enzymes related to its metabolism suggests a biological role for this polymer in platelets, in addition to the possibility of storage/mobilization of the polymeric form of glucose [4]. First, platelets contain the hexokinase enzyme (ADP: D-hexose-6-phosphotransferase, EC 2.7.1.1.), which catalyzes phosphorylation of glucose to glucose-6-phosphate in the first reaction of the glycolytic pathway [6]. Both the enzymes involved in converting glucose into its polymeric form and responsible for glycogen degradation, respectively glycogen synthase (GS) and glycogen phosphorylase (GP), are found in platelets [7]. The glyconeogenic pathway in platelets as well as fructose-1,6-diphosphatase activity were also described. Hence, platelets are able to synthesize glycogen from lactate, and the presence of fructose-1,6-diphosphatase allows them to produce glycogen from pyruvate or citrate [8].

The presence in platelets of enzymatic machinery related to glycogen metabolism shows that this energy resource is not merely a simple heritage from the megakaryocyte. Glycogen stores in human platelets are equivalent in amount, gram-to-gram, to those found in skeletal muscle [4], which is easily demonstrated by ultrastructure techniques, as well as by cytochemical assay [3]. However, despite the potential importance of glycogen determination in the platelets, a biochemical method to quantify this polymer in these cells is not available at the moment. The aim of our study was to establish a protocol to determine quantitatively the glycogen from human platelets.

\section{Material and Methods}

\section{Obtaining samples}

Human blood was collected from 16 healthy male volunteers registered as donors in the Hemotherapy Service of the Clinical Hospital of Porto Alegre, Rio Grande do Sul, Brazil. Two samples of 4 $\mathrm{mL}$ were taken from each donor from the antecubital vein and placed in vacutainers (BD Franklin Lakes, USA) containing $\mathrm{K}_{3}$-EDTA.

\section{Sample preparation}

Immediately after the sample was obtained, the two vacutainers from each subject were gently inverted 10 times and placed in a hematology analyzer ABX Micros ES 60 (HORIBA ABX SAS, Japan) to determine the number of platelets in each blood sample. Platelets were isolated as previously described by Mangano and Schwarcz [9]. The vacutainers were then centrifuged at $300 \mathrm{xg}$ for $5 \mathrm{~min}$ at $4^{\circ} \mathrm{C}$ and the platelet-rich plasma (PRP) was obtained. The supernatant (PRP) was carefully removed with a plastic tip, with care not to disturb the leukocyte layer. The volume of PRP collected from each sample was recorded and the PRP transferred to a microcentrifuge tube. The

*Corresponding author: Marcos Emílio Frizzo, Department of Morphological Sciences and Hemotherapy Service, Clinical Hospital of Porto Alegre, Universidade Federal do Rio Grande do Sul, Brazil, Tel: 5551 33084536; Fax: 555133083146 ; E-mail: frizzo@ufrgs.br

Received December 19, 2013; Accepted February 04, 2014; Published February 06, 2014

Citation: Rocha DS, Souza SK, Onsten TGH, da Silva RSM, Frizzo ME (2014) A Simple Method to Quantify Glycogen from Human Platelets. J Cytol Histol 5: 217. doi:10.4172/2157-7099.1000217

Copyright: (c) 2014 Rocha DS, et al. This is an open-access article distributed under the terms of the Creative Commons Attribution License, which permits unrestricted use, distribution, and reproduction in any medium, provided the original author and source are credited. 
PRP was then centrifuged at $7000 \times \mathrm{g}$ for $10 \mathrm{~min}$ at $4^{\circ} \mathrm{C}$. The plasma was discarded and the pellet resuspended in $0.5 \mathrm{~mL}$ of ice-cold 0.32 M-sucrose phosphate buffered ( $\mathrm{pH} 7.4$ ). The suspension, hereafter referred to as platelet concentrate (PC) was repeatedly passed through a plastic tip of $1 \mathrm{~mL}$ until the visible platelet aggregates were eliminated. An additional $0.5 \mathrm{~mL}$ of buffered sucrose was added to the suspension and the solution was mixed with 5 gentle inversions. The platelet suspension was again centrifuged at $7000 \times \mathrm{g}$ for $5 \mathrm{~min}$ at $4^{\circ} \mathrm{C}$. The supernatant was discarded and the platelet pellet was resuspended in a volume of sucrose $\left(0.32 \mathrm{M}, \mathrm{pH} 7.4\right.$ at $\left.4^{\circ} \mathrm{C}\right)$ equal to one-fifth of the initial volume of PRP obtained. After that, both PC suspensions obtained from each subject were blended and used for glycogen determination. The number of platelets was also determined in the platelet concentrate.

\section{Glycogen determination}

The assay to determine the glycogen content comprised the following steps: (1) cell lyses and glycogen extraction, (2) precipitation of glycogen and its washing, (3) conversion of glycogen to free glucose, (4) neutralization, and (5) detection of free glucose.

Step 1: In order to lyse the platelets, $0.25 \mathrm{~mL} \mathrm{KOH} \mathrm{(30 \% )} \mathrm{was} \mathrm{added}$ and the suspension was repeatedly (15 times) passed through a plastic tip of $1 \mathrm{~mL}$ to homogenize the mixture. The volume was transferred to a conical tube, and another $0.25 \mathrm{~mL} \mathrm{KOH} \mathrm{(30 \% )} \mathrm{was} \mathrm{added} \mathrm{and} \mathrm{the} \mathrm{mixture}$ homogenized again (15 times). For the glycogen extraction, the tube was boiled for $1 \mathrm{~h}$ and then $50 \mu \mathrm{L}$ of saturated $\mathrm{Na}_{2} \mathrm{SO}_{4}(35 \%)$ was added.

Step 2: The glycogen obtained from the sample was precipitated with $1 \mathrm{~mL}$ anhydrous ethanol and the tube placed briefly on a vortex mixer. After that, it was centrifuged at $750 \times \mathrm{g}$ for $10 \mathrm{~min}$, the supernatant carefully removed, and the precipitate resuspended in $0.5 \mathrm{~mL}$ of distilled water. The precipitation and washing steps were done once more, as described above, and the glycogen was finally resuspended in $0.3 \mathrm{~mL}$ of distilled water for its determination.

Step 3: The total glycogen obtained was converted to free glucose by acid hydrolysis. In each experiment, a standard curve (Figure 1) was established from a stock solution $\left(1 \mathrm{mg} \mathrm{mL}^{-1}\right)$ of glycogen type III (SIGMA, USA), and different amounts of glycogen, 50 (S1), 100 (S2), 150 (S3) and $200 \mu \mathrm{g}$ (S4) were used as a reference. The glycogen obtained from the sample and the one that was used in the standard curve were hydrolyzed with $\mathrm{HCl}(4 \mathrm{~N})$ by boiling for $1 \mathrm{~h}$. In this process, $0.3 \mathrm{~mL}$ of $\mathrm{HCl}$ was added to $\mathrm{S} 1, \mathrm{~S} 2, \mathrm{~S} 3, \mathrm{~S} 4$ and to the sample.

Step 4: After the mixture had cooled, $0.3 \mathrm{~mL}$ of $\mathrm{Na}_{2} \mathrm{CO}_{3}(2 \mathrm{M})$ was slowly added to each point of the standard curve and also to the sample to neutralize the solution. This process generates large amounts of bubbles and must be done carefully.

Step 5: After neutralization, free glucose obtained from hydrolysis of glycogen polymer of standards (S1-S4) and sample, was determined using a glucose-oxidase kit (LABORCLIN BIOLIQUID, Brazil). For this, an aliquot of $40 \mu \mathrm{L}$ of sample (or of the standard curve) was incubated with the enzyme reagent for $5 \mathrm{~min}$ at $37^{\circ} \mathrm{C}$. Absorbance was read at $505 \mathrm{~nm}$.

\section{Glycogen calculation}

The platelet number in each PC sample was used in the glycogen calculation. The equation used to calculate the amount of glycogen is given below:

$$
G=\frac{\left(\frac{1}{n} \sum_{i=1}^{n} \frac{G_{s n}}{A b s_{s n}}\right) \times A b s_{e} \times V}{N} \times 10^{6}
$$

where $\mathrm{G}=$ glycogen $\left(\mu \mathrm{g} / 10^{6}\right.$ platelets); $\mathrm{G}_{\mathrm{s}}=$ glycogen concentration standard $\left(\mu \mathrm{g} \quad \mathrm{mL}^{-1}\right) ; \quad A b s_{\mathrm{s}}=$ absorbance of glucose standard; $\mathrm{Abs}_{\mathrm{e}}=\mathrm{absorbance}$ of glucose from the sample; $\mathrm{V}=$ total volume of glycogen sample; $\mathrm{N}=$ total number of platelets.

\section{Results}

The cell count was performed in whole blood and again after obtaining the PC, using an automated hematology analyzer. The platelet number in each PC sample was used to normalize the glycogen quantity. Our data showed that platelet counting should be determined after the PC is obtained, since the cell number was reduced significantly during the process. Despite the loss of platelets, the mean platelet volume in the PC was exactly the same as measured in the whole blood, $7.8 \pm 0.7$ $\mu \mathrm{m}^{3}$ (mean $\pm \mathrm{SD}, \mathrm{n}=16$ ). In our analysis, the platelet number achieved in the PC was $1696 \pm 483 \times 10^{3} \mathrm{~mm}^{-3}($ mean $\pm S D, n=16)$ and showed an absence of contaminant cells.

After the platelet suspension was obtained, the cells were lysed and the glycogen was extracted through incubation with $\mathrm{KOH}$. The time chosen $(1 \mathrm{~h})$ was determined after tests of different exposure times (data not shown). The amount of glycogen quantified in human platelets was $0.263 \pm 0.101 \mu \mathrm{g} / 10^{6}$ platelets (mean $\pm S D, n=16$ ), and the data are shown in Figure 2.

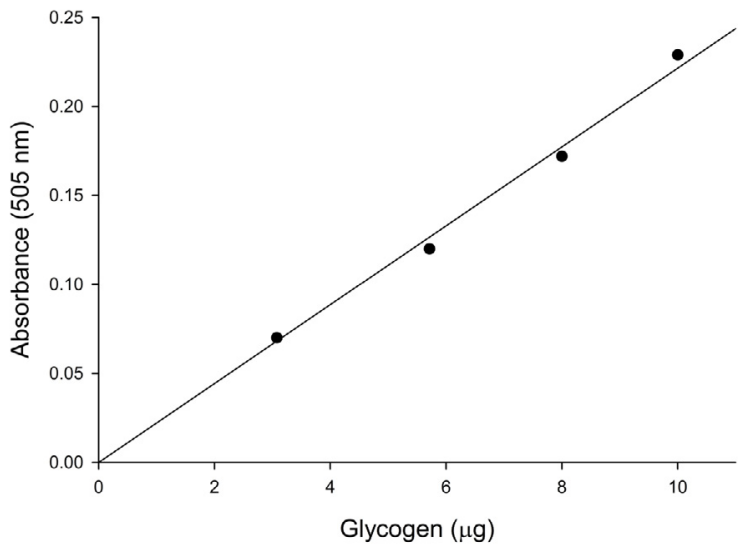

Figure 1: Representative standard curve for glycogen. In each experiment different amounts of glycogen (50,100,150 and $200 \mu \mathrm{g})$ were hydrolyzed. The values of glucose at each point were used to calculate the amount of glycogen in the sample $\left(R^{2}=0.99\right)$.

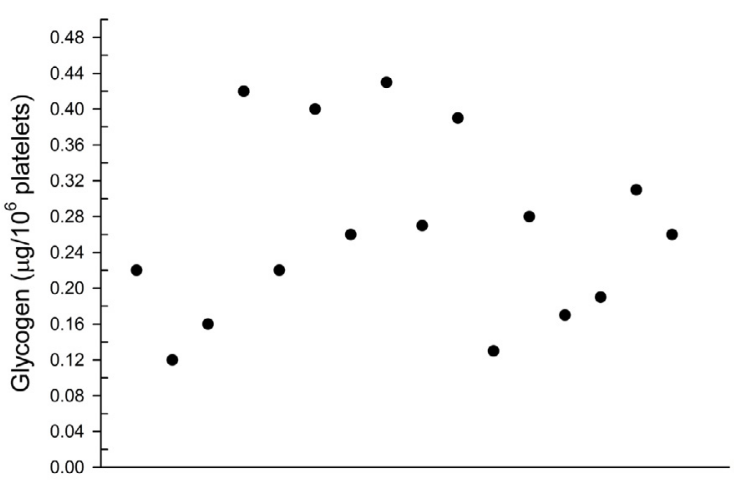

Figure 2: Scatter plot representing the values of glycogen obtained in the PC samples. The figure depicts the values of glycogen $(\mu \mathrm{g})$ from 16 healthy donors, normalized by the number of cells $\left(10^{6}\right.$ platelets). 
Citation: Rocha DS, Souza SK, Onsten TGH, da Silva RSM, Frizzo ME (2014) A Simple Method to Quantify Glycogen from Human Platelets. J Cytol Histol 5: 217. doi:10.4172/2157-7099.1000217

Page 3 of 3

\section{Discussion}

The large amount of glycogen and enzymatic machinery related to glycogen metabolism found in platelets suggest that this molecule plays a significant role. Nevertheless, few studies have examined platelet glycogen. The biological function of platelet glycogen seems not to be completely understood, especially considering that these cells may respond differently under different oxygen tensions [10,11]. Another important aspect is a potential relationship between platelet glycogen stores and physiopathological conditions [12-14]. The scarcity of data regarding glycogen function in platelets could be partially attributed to the lack of a protocol to quantify this molecule in these cells. The protocol described here allowed us to quantify the glycogen from human platelets, proving to be a simple and feasible method to measure these reserves from a blood sample. Even though it uses only small quantities of tissue, the method was rapid and reliable. Notably, the glycogen, determined from glucose, could also be normalized by other parameters besides counting the number of platelets; however, if used, this determination should be done in the PC and not in whole blood.

Recently, we hypothesized that the quantification of glycogen in platelets might be used as a peripheral biomarker of glycogen synthase kinase 3- $\beta$ (GSK3 $\beta$ ) activity [15], since this enzyme may directly regulate GS (to its inactive form) through phosphorylation [16]. In addition to its pivotal role in the regulation of glycogen synthesis through GS inactivation [17-19], GSK3 $\beta$ regulates several intracellular signaling pathways, including the cell cycle, gene expression and apoptosis [20,21]. This enzyme also has essential roles in neurons, where it regulates the cytoskeletal status [21,22], and abnormal regulation of its activity has been implicated in the pathophysiology of Alzheimer's disease [19], diabetes [14] and depression [23]. Although there is no evidence that glycogen levels is a biomarker of GSK3 activity, the determination of platelet glycogen might be useful to test this hypothesis, and this polymer could be a useful parameter to evaluate biochemical changes in other pathologies. With the protocol described here, we expect to contribute to future studies of platelet metabolism, especially regarding glycogen function in these cells.

\section{Acknowledgements}

This research was supported by grants from the Brazilian National Research Council (CNPq).

\section{References}

1. Murer EH (1968) Release reaction and energy metabolism in blood platelets with special reference to the burst in oxygen uptake. BiochimBiophysActa 162: 320-326.

2. White JG (1971) Platelet morphology: The circulating platelet. Academic Press, New York, USA.

3. Heckner F (1963) Polysaccharide in Blut- und Knochenmarkszellen: Zyto- und Histochemie in der Hämatologie. Springer-Verlag, Berlin, Germany.

4. Karpatkin S (1967) Studies on human platelet glycolysis: effect of glucose, cyanide, insulin, citrate, and agglutination and contraction on platelet glycolysis. J Clin Invest 46: 409-417.

5. White JG (1999) Platelet glycosomes. Platelets 10: 242-246.

6. Akkerman J-WN, Rijksen G, Gorter G, Staal GEJ (1984) Platelet functions and energy metabolism in a patient with hexokinase deficiency. Blood 63: 147-153.
7. Scott RB (1967) Activation of glycogen phosphorylase in blood platelets. Blood 30: $321-330$.

8. Karpatkin S, CharmatzA, Langer RM (1970) Glycogenesis and glyconeogenesis in human platelets. Incorporation of glucose, pyruvate, and citrate into platelet glycogen; glycogen synthetase and fructose-1,6-diphosphatase activity. J Clin Invest 49: 140-149.

9. Mangano RM, Schwarcz R (1981) The human platelet as a model for the glutamatergic neuron: platelet uptake of L-glutamate. J Neurochem 36: 10671076.

10. Doery JCG, Hirsh J, Cooper I (1970) Energy metabolism in human platelets: interrelationship between glycolysis and oxidative metabolism. Blood 36: 159168

11. Detwiler TC, Zivkovic RV (1970) Control of energy metabolism in platelets A comparison of aerobic and anaerobic metabolism in washed rat platelets. BiochimBiophysActa 197: 117-126.

12. Gunning W, Dole M, Brecher M, White JG (2013) The Medich giant platelet syndrome: two new cases. Platelets 24: 107-112.

13. Nakajima H, Raben N, Hamaguchi T, Yamasaki T (2002) Phosphofructokinase deficiency; past, present and future. CurrMol Med 2: 197-212.

14. Saltiel AR (2001) New perspectives into the molecular pathogenesis and treatment of type 2 diabetes. Cell 104: 517-529.

15. Frizzo ME (2013) Putative role of glycogen as a peripheral biomarker of GSK3 $\beta$ activity. Med Hypotheses 81: 376-378.

16. Rylatt DB, Aitken A, Bilham T, Condon GD, Embi N, Cohen P (1980) Glycogen synthase from rabbit skeletal muscle. Amino acid sequence at the sites phosphorylated by glycogen synthase kinase-3, and extension of the N-terminal sequence containing the site phosphorylated by phosphorylase kinase. Eur $\mathrm{J}$ Biochem107: 529-537.

17. Embi N, Rylatt DB, Cohen P (1980) Glycogen synthase kinase-3 from rabbit skeletal muscle. Separation from cyclic-AMP-dependent protein kinase and phosphorylase kinase. Eur J Biochem 107: 519-527.

18. Chin PC, Majdzadeh N, D'Mello SR (2005) Inhibition of GSK3b is a common event in neuroprotection by different survival factors. Brain Res Mol Brain Res 137: 193-201.

19. Balaraman Y, Limaye ER, Levey Al, Srinivasan S (2006) Glycogen synthase kinase $3 \mathrm{~B}$ and Alzheimer's disease: pathophysiological and therapeutic significance. Cell Mol Life Sci 63: 1226-1235.

20. Jope RS, Yuskaitis CJ, Beurel E (2007) Glycogen synthase kinase-3 (GSK3): inflammation, diseases, and therapeutics. Neurochem Res 32: 577-595.

21. Peineau S, Bradley C, Taghibiglou C, Doherty A, Bortolotto ZA, et al. (2008) The role of GSK-3 in synaptic plasticity. Br J Pharmacol 153: S428-437.

22. Grimes CA, Jope RS (2001) The multifaceted roles of glycogen synthase kinase 3beta in cellular signaling. ProgNeurobiol 65: 391-426.

23. Joaquim HPG, Talib LL, Forlenza OV, Diniz BS, Gattaz WF (2012) Long-term sertraline treatment increases expression and decreases phosphorylation of glycogen synthase kinase-3B in platelets of patients with late-life major depression. J Psychiatr Res 46: 1053-1058. 